authentic material available for research. This can only be done by inducing collectors to obtain wood samples from the trunks of the same trees from which herbarium material is taken. It should be our aim to build up wood collections comparable to our national herbaria; those that we have are hopelessly encumbered by com. mercial samples of timbers and specimens col- lected in all good faith but entirely without regard to the need of ensuring their true botanical authenticity. There can be no better example of the need for co-operative effort than the preliminary work necessary to establish on a sound basis the thorough investigation and successful utilisation of the timber resources of the overseas dominions of the British Empire.

\title{
Stellar Radial Velocities
}

\section{By Dr. R. O. ReDman}

THERE has recently been published by the Lick Observatory a General Catalogue of the Radial Velocities of Stars, Nebulæ, and Clusters by Joseph Haines Moore.* This gives all radial velocities published up to January 1, 1932, and includes 6739 stars, 133 gaseous nebulæ, 18 globular clusters and 90 extra-galactic nebulæ. For some time a need has been felt for such a compilation, and after consultation with the members of the radial velocity commission of the International Astronomical Union, which includes practically all the workers active in this field, Dr. Moore undertook the task. Himself a radial velocity observer of considerable experience, he has carried out this work very thoroughly and the publication forms a compact collection of observational data of extremely great value.

It is very fitting that this catalogue should be issued by the Lick Observatory, which has played. such a leading part during the past forty years in the measurement of radial velocities of stars and nebulæ. The first attack on the measurement of the Doppler shift in stellar spectra was made by Huggins in 1866, using visual methods, but the task proved difficult, and reasonably reliable results were not obtained until Keeler's work with the Lick 36-inch refractor about 1890. Even then velocities were obtained only with one or two of the brightest stars in the sky.

However, at about this same time, line of sight velocities were successfully measured at Potsdam by photography. The great superiority of the photographic method was quickly realised and soon extensive work was started, particularly by Campbell and his associates at the Lick Observatory. Observations have been accumulating with increasing rapidity ever since. As is well known, the results have been among the most valuable of astrophysical data and have found many applications, in the determination of masses in binary stars, in the study of variable stars, in stellar motions and the dynamics of the Milky Way system. Radial velocity technique has also contributed support to relativity theory in observations of Sirius B and to the theory of the expanding universe in recent observations of the spiral nebulæ.

The practical problem of radial velocity determination consists in the measurement of displacements of lines in an absorption spectrum

* Publications of the Lick Observatory, Volume 18, 1932. No. 3292, Vol. 130] formed by light from an extremely faint source. The unavoidable complications present in all astronomical observations, namely a rotating earth and an unsteady atmosphere of varying transparency, have also to be contended with. The average stellar velocity in the line of sight is around 15 or $20 \mathrm{~km}$./sec., which corresponds in the ordinary photographic region of the spectrum to a Doppler shift of approximately one quarter of an angstrom.

The experience of the past thirty or forty years has indicated with little ambiguity the most desirable type of instrument to use. A telescope of large aperture is essential- -36 in. is the least size with which any important amount of work is done at present-and, although refractors have in several cases given excellent service, it is generally considered that reflectors are more desirable. Their advantages lie in ease of construction, in their perfect achromatism, in their greater efficiency in the region of shorter wavelengths and in the long equivalent focal length which may be obtained without an inconveniently long telescope tube. The Cassegrain arrargement of mirrors, with a convex secondary reflecting the beam back through a hole in the centre of the primary, is undoubtedly the best.

The spectrograph is normally carried on the telescope, and since it may then be required to work in almost any position, it has to be constructed and mounted with special precautions against flexure or distortion of any kind. Prisms are usually employed since they are less wasteful of light than are diffraction gratings. One prism is most commonly used, although the number may be two or three for the brighter stars, and several interchangeable cameras of various focal lengths are usually available. In this way a fairly wide range of dispersions can be provided, varying perhaps from near 10 to around 100 angstroms per millimetre at $\mathrm{H}_{\gamma}(\lambda$ 4340) and suitable for use with stars of a wide range of apparent brightness. Since effectively the observing is done in the open air, the spectrograph should be enclosed in an insulating case and its temperature controlled by a thermostat. Exposure times vary enormously with magnitude and type of stars, with observing conditions and with the particular instrument used. Extensive radial velocity work is rarely carried out where more than one or two hours per photograph are required in fair weather. The 
resulting photographs are measured on special micrometer machines, the positions of the spectral lines being determined with reference to a comparison spectrum of the iron are or some other convenient standard source.

Particular reference may be made to the work on extra-galactic nebulæ, the spectra of which almost without exception show displacements corresponding to large velocities of recession. The objects in question are generally extremely faint, and in addition their light is spread over an appreciable area of the sky, instead of being concentrated like that of a star. They are more difficult to observe than the diffuse and planetary nebulæ, since they have absorption, not emission, spectra. Pioneer work was done by V. M. Slipher at the Lowell Observatory, Flagstaff, but the more extensive recent measurements have been made at Mt. Wilson, where spectra of extremely small dispersion are taken with a special short focus camera lens working at $f 0 \cdot 6$. The slit is used very wide and the exposures are sometimes very long, extending over several nights. In the photographs it is generally possible to distinguish only one or two of the strongest features of the spectrum, but the displacements, thanks to their great size, are usually determined well within ten per cent.

Reverting to more normal stellar work, the errors of measurement of radial velocities, as one might expect, vary a great deal according to the dispersion of the spectra and the nature of the spectrum lines. With a one prism instrument giving 25 or $30 \mathrm{~A} . / \mathrm{mm}$. at $\mathrm{H}_{\gamma}$, the probable error per plate is approximately $1 \mathrm{~km}$./ sec. in the case of a star with many sharp spectral lines, but with some spectra where the lines are few and nebulous, this error may easily exceed $5 \mathrm{~km}$./sec. Systematic errors have in the past usually been evaluated by intercomparison of results from different observatories. Their average value is generally rather less than $1 \mathrm{~km}$. $/$ sec. Now, however, as work is being pushed further to fainter apparent magnitudes, there is considerably less overlap than formerly between the work at one observatory and another, and standard stars are coming into use for the purpose of checking systematic errors. Alist of such stars was published some years ago by the radial velocity commission of the International Astronomical Union and has proved very valuable. It is at present being revised and extended to fainter stars.

An in'spection of Dr. Moore's catalogue gives some idea of the total progress made to date in this work. Thanks chiefly to the Lick Observatory, all stars have been adequately observed down to magnitude 5.51, but apart from this no group of stars has been completely observed over both northern and southern hemispheres. In the northern sky various groups of objects such as the Boss stars, the brighter dwarfs, the O to B5 stars down to magnitude $7 \cdot 5$, and certain classes of variables, have been observed, and further systematic work is being pushed forward, particularly at the Lick, Victoria and Mt. Wilson observatories. On the other hand, the southern sky below declination $-25^{\circ}$, with the exception of the brighter stars just mentioned, remains practically untouched. Neglecting for the moment the fact that there are known to be many variable and peculiar stars of great interest in this region, knowledge of which would be very greatly advanced by radial velocity observations, this gap in our data is most serious in work on stellar motions. Until it is filled, observational studies of such question's as galactic rotation and certain other features of stellar movements must necessarily be incomplete.

Unfortunately, there seems to be no immediate certainty of a suitable telescope being established in the southern hemisphere for this purpose. At the recent meetings at Cambridge, Massachusetts, the radial velocity commission and later the general assembly of the International Astronomical Union, fully alive to the unsatisfactory nature of the present situation, passed a resolution asking that every effort be made to further any project aiming at the establishment and efficient working in the southern hemisphere of a large reflecting telescope for stellar spectroscopy and in particular for the determination of radial velocities. It seems unfortunate that some of the large sums of money devoted to several schemes at present on foot to build large instruments in the northern hemisphere, could not have been diverted towards filling this need in the southern sky.

Apart from this gap, which will have to be filled at some time, future work in radial velocities is likely to concentrate on refined observations of the brightest stars, on variables and peculiar objects, and on obtaining large numbers of velocities of fainter stars for statistical work. These last need not be of very great accuracy, although systematic errors will have to be carefully watched. There is in these three directions an almost unlimited field for large telescopes.

\section{Obituary}

Mr. Frank Finn

GRANK FINN, whose recent death, at sixtyfour years of age, we regret to record, was an exhibitioner of Brasenose College, Oxford, and an excellent classical scholar. Whilst at Oxford he took a great interest in ornithology, both as an observer of birds and a systematic naturalist. $\mathrm{He}$ acquired a reputation amongst dealers and spent a great deal of his time in identifying collections of skins. This pursuit, unfortunately, occupied much of the time that he should have devoted to classics, with the result that his academic success by no means equalled his capacity. It may be said that this showed almost the leading defect in his character, which prevented him from being really successful in any of his undertakings. He

No. 3292, Vol. 130] 